\title{
Inactivation of DNA-PK by knockdown DNA-PKes or NU7441 impairs non-homologous end-joining of radiation-induced double strand break repair
}

\author{
JUN DONG $^{1 *}$, YUFENG REN $^{1 *}$, TIAN ZHANG $^{1 *}$, ZHENYU WANG ${ }^{1}$, CLIFTON C. LING $^{2}$, \\ GLORIA C. $\mathrm{LI}^{2}$, FUQIU HE ${ }^{2}$, CHENGTAO WANG ${ }^{1}$ and BIXIU WEN ${ }^{1,2}$ \\ ${ }^{1}$ Department of Radiation Oncology, The First Affiliated Hospital, Sun Yat-sen University, Guangzhou, \\ Guangdong 510080, P.R. China; ${ }^{2}$ Department of Medical Physics and Radiation Oncology, \\ Memorial Sloan-Kettering Cancer Center, New York, NY 10021, USA
}

Received June 15, 2017; Accepted December 20, 2017

DOI: $10.3892 /$ or.2018.6217

\begin{abstract}
The DNA-dependent protein kinase (DNA-PK) complex plays a pivotal role in non-homologous end-joining (NHEJ) repair. We investigated the mechanism of NU7441, a highly selective DNA-PK inhibitor, in NHEJ-competent mouse embryonic fibroblast (MEF) cells and NHEJ-deficient cells and explored the feasibility of its application in radiosensitizing nasopharyngeal carcinoma (NPC) cells. We generated wild-type and DNA-PKcs ${ }^{-/} \mathrm{MEF}$ cells. Clonogenic survival assays, flow cytometry, and immunoblotting were performed to study the effect of NU7441 on survival, cell cycle, and DNA repair. NU7441 profoundly radiosensitized wild-type MEF cells and SUNE-1 cells, but not DNA-PKcs ${ }^{-/}$MEF cells. NU7441 significantly suppressed radiation-induced DSB repair post-irradiation through unrepaired and lethal DNA damage, the cell cycle arrest. The effect was associated with the activa-
\end{abstract}

Correspondence to: Professor Bixiu Wen or Dr Chengtao Wang, Department of Radiation Oncology, The First Affiliated Hospital, Sun Yat-sen University, 58 Zhongshan II Road, Guangzhou, Guangdong 510080, P.R. China

E-mail: wenbix@mail.sysu.edu.cn

E-mail: wangcht5@mail.sysu.edu.cn

Abbreviations: DSB, DNA double strand breaks; IR, ionizing radiation; NHEJ, non-homologous end-joining; HR, homologous recombination; DNA-PKcs, DNA-dependent protein kinase catalytic subunit; MEF, mouse embryonic fibroblast; WT, wild-type; DDR, DNA damage response; ATR, ataxia-telangiectasia and Rad3-related; ATM, ataxia-telangiectasia mutated; CHK, checkpoint effector kinase; NPC, nasopharyngeal carcinoma; FBS, fetal bovine serum; DMSO, dissolved in dimethylsulfoxide; DAPI, 4,6-diamidino-2-phenylindole; FACS, fluorescence-activated cell sorting; PI, propidium iodide; DNKu70, dominant negative fragment $\mathrm{Ku} 70$

Key words: irradiation, DNA-PK, non-homologous end-joining, double strand break, NU7441, nasopharyngeal carcinoma tion of cell cycle checkpoints. The present study revealed a mechanism by which inhibition of DNA-PK sensitizes cells to irradiation suggesting that radiotherapy in combination with DNA-PK inhibitor is a promising paradigm for the management of NPC which merits further investigation.

\section{Introduction}

DNA double strand breaks (DSBs) generated by ionizing radiation (IR) and genotoxic agents must be repaired to protect genetic fidelity. When mammalian cells are exposed to IR, several physiological processes are invoked, including DSB repair, cell cycle checkpoints, apoptosis, autophagy and telomere-DSB fusion. DSBs may: i) be repaired during the G2/M phase and enter another cell cycle; ii) become apoptotic; or iii) repopulate with aberrant genome. DSBs are repaired by two main pathways: non-homologous end-joining (NHEJ) and homologous recombination (HR) $(1,2)$. The modalities to detect DNA double strand breaks (DSBs) include immunofluorescent staining of $\gamma \mathrm{H} 2 \mathrm{AX}$ and FACS analysis of G2/M arrest in the cell cycle.

DNA-dependent protein kinase catalytic subunit (DNA-PKcs) is a crucial factor of NHEJ. Our previous studies and other research have demonstrated that mouse cells and human cancer cells deficient in the DNA-PK complex, which is composed of $\mathrm{Ku} 70 / \mathrm{Ku} 80$ heterodimer and DNA-PKcs, are hypersensitive to IR (3-5). The DNA-PK complex contributes to early stage damage-induced cell cycle arrest and DNA repair (6). Thus, DNA-PK may be a valid target for radiotherapeutic intervention in cancer therapy.

The cell cycle is a decisive factor in the choice of DSB repair pathway. NHEJ is favored in the G1 phase and HR is active in the $\mathrm{S}$ and $\mathrm{G} 2$ phases (7). SCID cells defective in DNA-PKcs are hypersensitive to X-ray during G1 and early $\mathrm{S}$ phase (8). Our previous research demonstrated that cell cycle arrest in the $\mathrm{G} 2$ phase is more common in $\mathrm{Ku} 70^{-1}$ and $\mathrm{Ku} 80^{-/}$mouse embryonic fibroblast (MEF) cells than wild-type (WT) MEF cells at $6 \mathrm{~h}$ post-IR (9). Inactivation of DNA-PK results in prolonged G2/M phase arrest in $\mathrm{ATM}^{-/-}$ human fibroblasts (10). 
DNA damage response (DDR) proteins, including ataxiatelangiectasia and Rad3-related (ATR), ataxia-telangiectasia mutated (ATM), checkpoint effector kinase 1 (CHK1), CHK2, and DNA-PKcs, form a phalanx of kinases in response to DSB. ATM and ATR trigger the phosphorylation of CHK2 and CHK1, respectively (11). The ATM-CHK2 and ATR-CHK1 pathways collaboratively modulate cell cycle arrest $(12,13)$. Akt has been reported to be activated in a DNA-PK-dependent manner at the site of DSBs and to promote NHEJ via DNA-PK activation $(1,14)$.

Strategies for improving the efficacy of conventional radiotherapy with agents inducing DSB repair deficiency are feasible by better understanding the interconnectivity between the diverse molecular mechanisms. NU7441, a highly potent and selective DNA-PK inhibitor, has been reported to effectively radiosensitize several human cancer cells (15-22).

Nasopharyngeal carcinoma (NPC), an endemic cancer in southern China, has been cured by radiation therapy in combination with concurrent chemotherapy. The 5-year survival rate can be expected to reach $75 \%$; whereas local tumor recurrence remains one of the main obstacles for successful treatment, especially for those with locally advanced NPC (23). Novel agents have been found to enhance radiosensitivity to decrease local recurrence in NPC patients who are treated with radiation therapy in combination with targeted therapy. The application of DNA-PK inhibitor to enhance radiosensitivity may improve treatment outcomes for this cohort of patients with NPC. However, the cellular specificity and mechanism of DNA-PK inhibition-mediated antitumor activity is still unclear.

Comparing DNA-PKcs-deficient and DNA-PKcscompetent cells, we explored the mechanism of NHEJ repair involving cell cycle checkpoints and the potential synergistic effect of IR and DNA-PK inhibitor. We further corroborate the synergistic effect of DNA-PK inhibition on enhanced radiosensitivity in human NPC cells.

\section{Materials and methods}

Cell lines and treatments. DNA-PKcs ${ }^{-/}$and WT MEF cells were kindly provided by Professor Gloria C. Li from Memorial Sloan-Kettering Cancer Center, USA $(3,24,25)$. SUNE-1 cell line, derived from a patient with undifferentiated NPC $(26,27)$, was a gift from Professor Tiebang Kang at Sun Yat-sen University Cancer Center. WT, DNA-PKcs ${ }^{-/-} \mathrm{MEF}$ cells and human NPC SUNE-1 cells were maintained in RPMI-1640 supplemented with $10 \%$ fetal bovine serum (FBS), penicillin, and streptomycin at $37^{\circ} \mathrm{C}$ in $5 \% \mathrm{CO}_{2}$.

The DNA-PK inhibitor NU7441 (Tocris Bioscience, Bristol, UK) was dissolved in dimethylsulfoxide (DMSO) as a $5 \mathrm{mmol} / 1$ stock solution and stored at $-20^{\circ} \mathrm{C}$. All drugs were added to cells with a final DMSO concentration of $0.5 \%$. Cells were exposed to X-rays generated by a Rad Source RS2000 irradiator (Rad Source Technologies, Buford, GA, USA) operating at $25 \mathrm{~mA}$ with a $0.3 \mathrm{~mm}$ Al filter and effective photon energy of $160 \mathrm{kV}$. The dose rate at an irradiation distance of $48.6 \mathrm{~cm}$ was $1.31 \mathrm{~Gy} / \mathrm{min}$.

Clonogenic survival assays. Clonogenic cell survival was determined by the colony formation assay as described previously (28). A total of $1.5 \times 10^{5}$ exponentially growing WT, DNA-PKcs ${ }^{-/}$MEF cells and SUNE-1 cells were supplemented with control or NU7441 $(1 \mu \mathrm{M})$-containing medium for $1 \mathrm{~h}$ and then exposed to IR at the indicated dose. After IR, the cells were incubated with or without NU7441 for a further $24 \mathrm{~h}$. Cells were harvested and plated in drug-free medium at 20-200 colonies per 100-mm dish in triplicate and left to develop colonies. Approximately 7-10 days later, the medium was discarded and the colonies were fixed and stained with crystal violet. Colonies consisting of $>50$ cells were counted. Surviving fractions were normalized by the plating efficiency of unirradiated controls.

Immunofluorescence microscopy foci assay. Cells were exposed to IR of $5 \mathrm{~Gy}$ in the presence or absence of $2 \mu \mathrm{M}$ NU7441 $1 \mathrm{~h}$ pre-IR and collected at the indicated time. Cells were fixed with $4 \%$ paraformaldehyde, permeabilized with $0.2 \%$ Triton $\mathrm{X}-100$, and blocked with $3 \%$ bovine serum albumin. The cover slips were incubated with anti- $\gamma \mathrm{H} 2 \mathrm{AX}$ antibody (1:200; Millipore, Billerica, MA, USA) overnight at $4^{\circ} \mathrm{C}$, followed by incubation with conjugated secondary antibody for $1.5 \mathrm{~h}$ in the dark. Nuclei were stained with 4,6-diamidino2-phenylindole (DAPI; Roche, Basel, Switzerland). Images were captured using an LSM 710 confocal microscope (Zeiss, Oberkochen, Germany), with foci counted in 30 cells. Quantitative image analysis was performed by ImageJ (29).

Fluorescence-activated cell sorting (FACS). To detect the percentage of cells with $\gamma \mathrm{H} 2 \mathrm{AX}$, WT MEF cells were incubated with FBS-free medium to synchronize them into G1 phase. Twelve hours later, the FBS-free medium was removed and cells recovered in medium containing $10 \%$ FBS for another $24 \mathrm{~h}$. The cells were irradiated with $5 \mathrm{~Gy}$ alone or in combination with $2 \mu \mathrm{M}$ NU7441. Following treatment, the cells were trypsinized at the indicated time, fixed, treated with $0.2 \%$ Triton X-100, and blocked in $3 \%$ BSA. The cells were stained with anti- $\gamma \mathrm{H} 2 \mathrm{AX}$ antibody (1:100; Cell Signaling Technology, Inc., Danvers, MA, USA) and DAPI. A minimum of 10,000 labeled cells were acquired and analyzed by FlowJo 7.6.1 software (30).

For cell cycle analysis, SUNE-1 cells were exposed to irradiation of 5-Gy in the presence or absence of $2 \mu \mathrm{M}$ NU7441. Cells were collected at indicated time post-IR and fixed with $70 \%$ ethanol. Cellular DNA was labeled with propidium iodide (PI). The cell cycle distribution was determined with 10,000 cells using FACS flow cytometry (Gallios; Beckman Coulter, Inc., Brea, CA, USA). The proportion of cells in different phases was gated and calculated using Flowjo 7.6.1 software.

Western blot analysis. Total protein and phosphorylated protein levels were analyzed by western blot analysis. Briefly, following treatment, cells were lysed and whole-cell lysates resolved by SDS-PAGE (6 or 10\%) and immunoblotted with the indicated antibodies. The following primary antibodies were used for immunoblotting: anti-AKT, anti-phosphoAkt (S473), anti-CHK1, anti-phospho-CHK1 (S345), anti-CHK2, anti-ATR, anti-phospho-ATR (S428), antiATM, anti-rabbit/mouse horseradish peroxidase-conjugated secondary antibodies (Cell Signaling Technology, Inc.), 

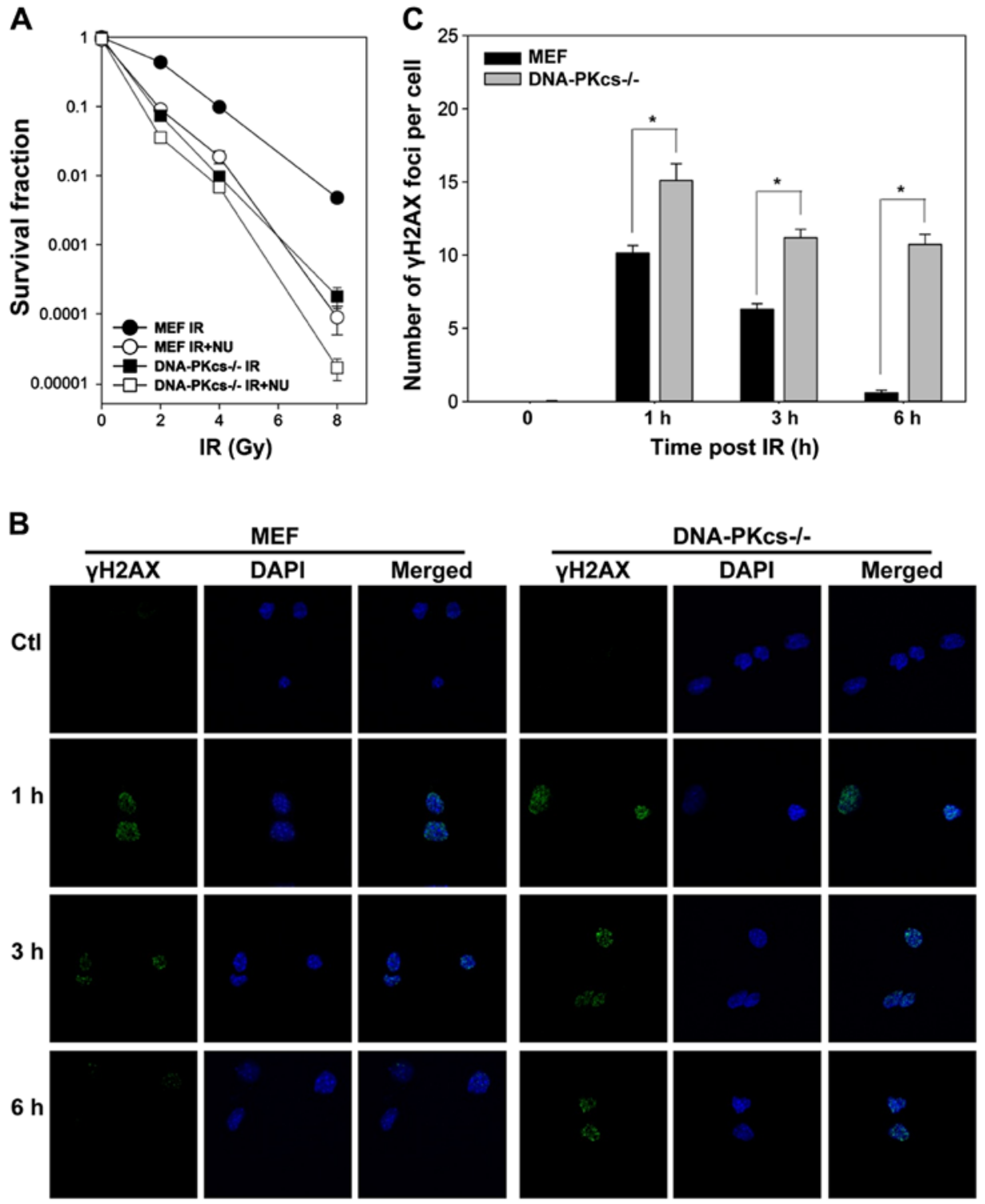

Figure 1. DNA-PKcs-dependent clonogenic survival and $\gamma \mathrm{H} 2 \mathrm{AX}$ foci formation after ionizing radiation (IR). (A) WT and DNA-PKcs ${ }^{-/}$MEF cells were exposed to IR alone or in the presence of NU7441 $(1 \mu \mathrm{M})$. Clonogenic survival data are represented as the mean \pm SD. (B) Cells were irradiated at 5 Gy and stained with $\gamma \mathrm{H} 2 \mathrm{AX}$ antibodies and DAPI at the indicated times. (C) Quantification of $\gamma \mathrm{H} 2 \mathrm{AX}$ foci formation in (B). Representative photomicrographs (x1,000 magnification) are shown. Data are presented as mean \pm SEM. ${ }^{*} \mathrm{p}<0.05$, Student's t-test.

anti-Ku70, anti-phospho-CHK2 (T68) (Novus Biologicals LLC, Littleton, CO, USA), anti-phospho-ATM (S1981), antiRAD51 (Santa Cruz Biotechnology, Inc., Santa Cruz, CA, USA), and anti- $\beta$-actin (Millipore).

Statistical analysis. The data are presented as mean \pm SD of at least three independent experiments. The significance of G2/M phase arrest between IR and IR and NU7441 was determined by ANOVA and all other statistical tests were performed using Student's t-test in Sigma Plot 12.5. Significance was defined as p-values of $<0.05$.

\section{Results}

Enhancing NHEJ pathway-mediated radiosensitivity and increasing radiation-induced $\gamma H 2 A X$ foci by suppressing $D N A-P K$ activity. To investigate the role of DNA-PK in response to IR, we utilized WT and DNA-PKcs ${ }^{-/-} \mathrm{MEF}$ cells to determine differences in radiosensitivity in colony formation assay. Irradiation reduced cell survival in DNA-PKcs ${ }^{-/} \mathrm{MEF}$ cells. NU7441 $(1 \mu \mathrm{mol} / \mathrm{l})$ significantly enhanced radiosensitivity in WT MEF cells, whereas a modest effect was observed in DNA-PKcs ${ }^{-/}$MEF cells (Fig. 1A), supporting that potentiation was attributable to the inhibition of the DNA-PK complex involving in DNA-PKcs gene expression.

We assessed the capacity of DSB repair by counting cellular $\gamma \mathrm{H} 2 \mathrm{AX}$ foci, which is routinely performed to assess the amount of DSBs (31). As shown in Fig. 1B and C, IR led to a rapid peak of $\gamma \mathrm{H} 2 \mathrm{AX}$ foci formation (within $1 \mathrm{~h}$ ). $\gamma \mathrm{H} 2 \mathrm{AX}$ foci in WT MEF cells gradually resolved to the baseline level at $6 \mathrm{~h}$ post-IR but declined with much slower kinetics in DNA-PKcs ${ }^{-/-} \mathrm{MEF}$ cells. Notably, we observed considerable residual $\gamma \mathrm{H} 2 \mathrm{AX}$ foci in DNA-PKcs ${ }^{-/-} \mathrm{MEF}$ cells compared to WT MEF cells at 1, 3, and $6 \mathrm{~h}$ after treatment (Fig. 1C), indicating that attenuation of the NHEJ pathway impedes DSB repair with highly significant radiosensitivity. 
A
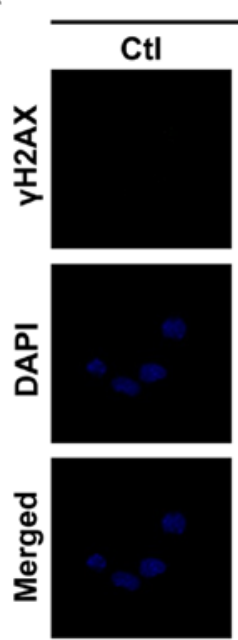

B

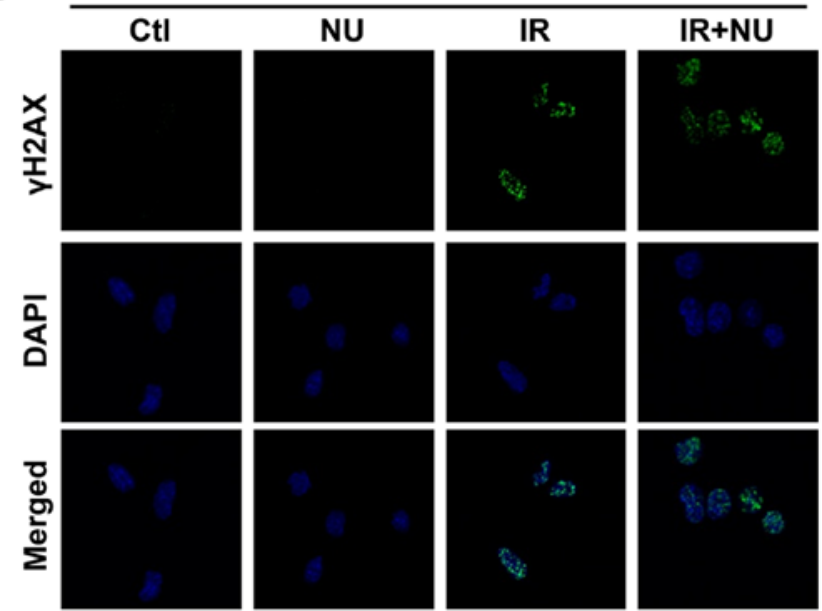

C

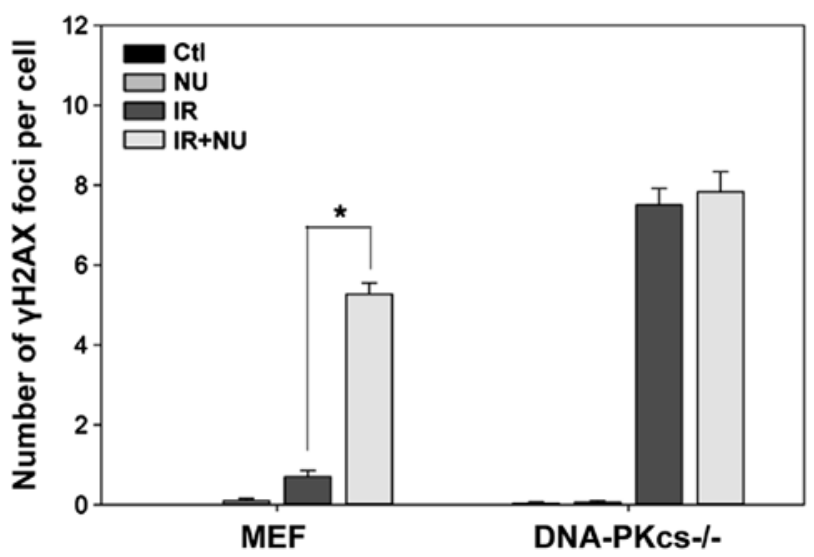

Figure 2. $\gamma \mathrm{H} 2 \mathrm{AX}$ foci formation and its quantification analysis following exposure to ionizing radiation (IR) in the presence or absence of NU7441. (A) WT and (B) DNA-PKcs ${ }^{-/} \mathrm{MEF}$ cells were immunostained with anti- $\gamma \mathrm{H} 2 \mathrm{AX}$ antibody $6 \mathrm{~h}$ post-IR $(5 \mathrm{~Gy})$ in the absence of presence of NU7441 $(2 \mu \mathrm{M})$. Representative photomicrographs (x1,000 magnification) are shown. (C) Quantification of $\gamma \mathrm{H} 2 \mathrm{AX}$ foci. Data are presented as mean $\pm \mathrm{SEM}$. ${ }^{*} \mathrm{p}<0.05$, Student's t-test.

Effect of NU7441 on radiation-induced DSB repair. Based on our finding that DSBs are almost completely repaired in WT MEF cells but persist in DNA-PKcs ${ }^{-/-}$MEF cells at $6 \mathrm{~h}$ post-IR, we evaluated the effect of NU7441 on DSBs at $6 \mathrm{~h}$ after irradiation. We detected a striking accumulation of $\gamma \mathrm{H} 2 \mathrm{AX}$ foci $(5.3 \pm 0.3$ foci per cell) in WT MEF cells treated with both IR and NU7441 compared to cells treated with IR alone $(0.7 \pm 0.2$ foci per cell; Fig. $2 \mathrm{~A}$ and C). NU7441 moderately affected the level of radiation-induced $\gamma \mathrm{H} 2 \mathrm{AX}$ foci in DNA-PKcs ${ }^{-/}$MEF cells (Fig. 2B and C). In contrast, treatment with NU7441 alone had a negligible effect on $\gamma \mathrm{H} 2 \mathrm{AX}$ foci formation (Fig. 2). These results were mirrored by a corresponding decrease in viability (as measured by clonogenic survival assays).

Effect of NU7441 on crosstalk between DSBs and cell cycle. In line with our previous data, deficiency of $\mathrm{Ku}$ heterodimer blocks cell cycle progression (9). NU7441 is designed to target the DNA-PK complex and may not only impair DSB repair, but also influence the cell cycle profile. In synchronized WT MEF cells, combined treatment with NU7441 and IR resulted in more $\gamma \mathrm{H} 2 \mathrm{AX}$-positive cells than IR alone. The maximum level of $\gamma \mathrm{H} 2 \mathrm{AX}$ expression was detected at $3 \mathrm{~h}$ after combined treatment (Fig. 3A and B). Quantification of the percentage of cells with $\gamma \mathrm{H} 2 \mathrm{AX}$ in the G2/M phase showed a slight decrease $6 \mathrm{~h}$ after combined treatment (Fig. 3C).

Mobilization of DDR proteins following exposure to IR. Because IR-induced DSBs activate the DDR and amplify the cellular damage signal by phosphorylating cell cycle checkpoints (32), we assessed the role of NU7441 in the activation of DDR-related gene expression. Treatment with NU7441 was associated with a significant increase in phospho-CHK1 levels in response to radiation (Fig. 4A). Combined treatment with NU7441 and IR resulted in the inhibition of CHK2 phosphorylation compared to treatment with IR alone (Fig. 4B). IR-induced Akt activity was inhibited by NU7441 only in WT MEF cells (Fig. 4C). NU7441 potently inhibited the expression of RAD51, a marker of the HR pathway, suggesting that it may be an efficient inhibitor targeting both the NHEJ and HR repair pathways (Fig. 4C).

Enhancement of antitumor activity with the addition of NU7441 to IR in SUNE-1 cells. Clonogenic survival assays revealed that NU7441 markedly increased the cytotoxicity of IR (Fig. 5A). Treatment with IR and NU7441 resulted in more pronounced DSBs compared to treatment with IR alone (Fig. 5B and C) at $6 \mathrm{~h}$ or $12 \mathrm{~h}$ post-IR, whereas NU7441 alone did not affect $\gamma \mathrm{H} 2 \mathrm{AX}$ foci formation.

Our previous study showed that NU7441 alone did not induce cytotoxicity and DSB (25). Combination of IR and NU7441 significantly enhances radiosensitivity and induces more DSB than IR alone. It plays an important role in radiosensitizing cells but treatment of NU7441 alone might not influence the cell cycle progression. We aimed to compare the difference of cell cycle distribution between IR alone and IR + NU7441.

Irradiated cells undergo abnormal cell cycle progression in the absence of a proficient NHEJ factor (33). Cells are more sensitive to radiation in the $\mathrm{G} 2 / \mathrm{M}$ phase of cell cycle than any other phases (34). We hypothesized that the addition of NU7441 would augment radiation-induced accumulation of SUNE-1 cells in G2 phase. As expected, the G2/M arrest was observed in SUNE-1 cells when exposed to IR with or without NU7441. The difference between the groups of IR alone and 
A
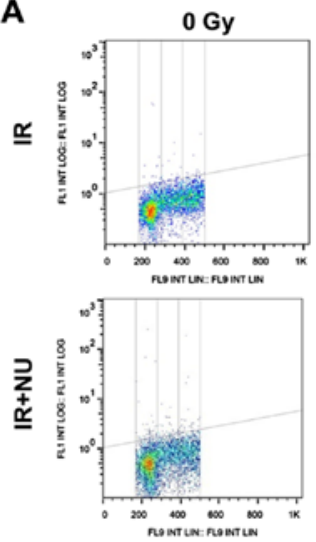

B

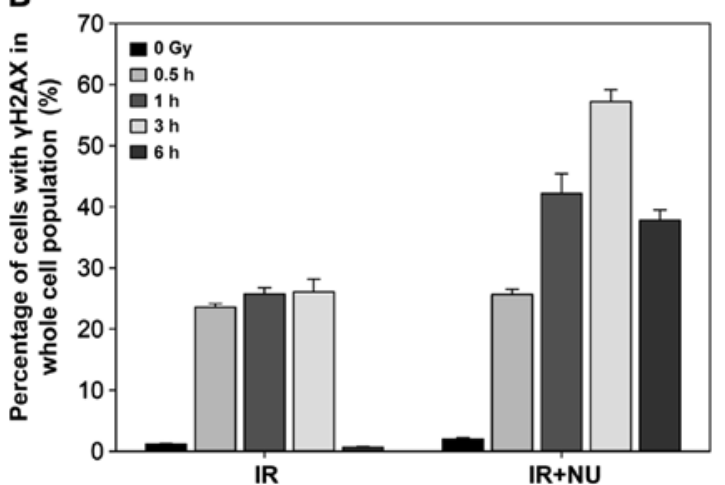

$1 \mathrm{~h}$
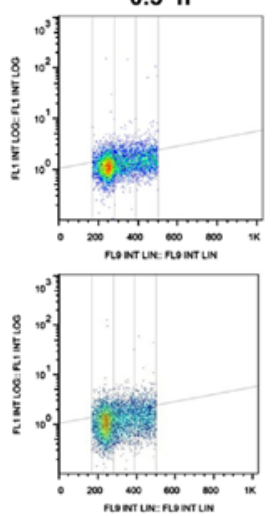

$3 \mathrm{~h}$
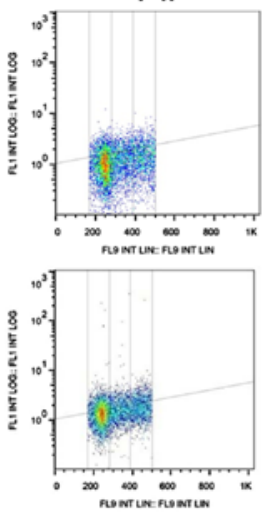
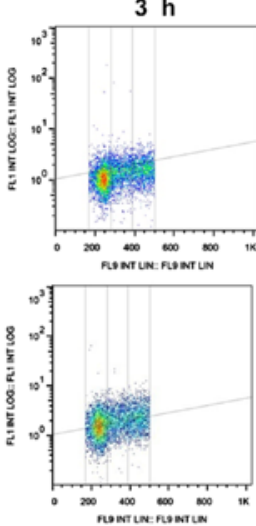

$6 \mathrm{~h}$
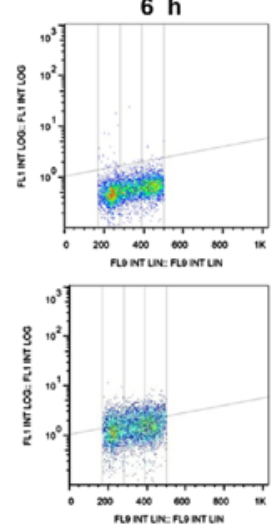

C

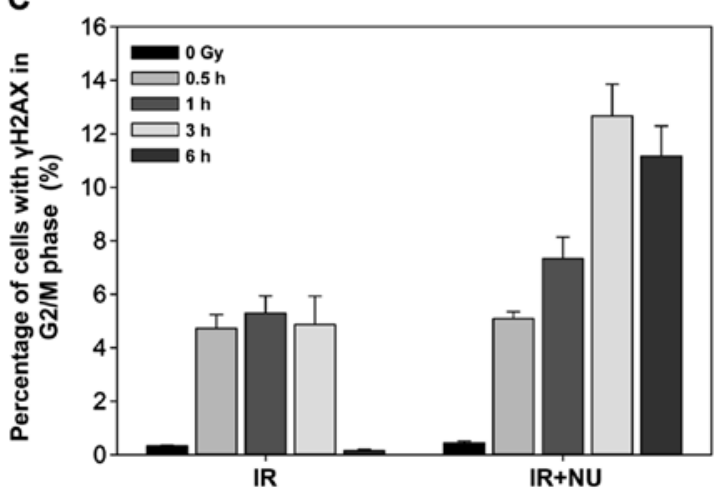

Figure 3. Interaction between double strand break repair and cell cycle in MEF cells exposed to ionizing radiation (IR) in the presence or absence of NU7441. $\gamma \mathrm{H} 2 \mathrm{AX}$ was induced by 5-Gy X-ray alone or combination with $2 \mu \mathrm{M}$ NU7441 in MEF cells. The cells were co-stained with DAPI and $\gamma \mathrm{H} 2 \mathrm{AX}$ antibody. (A) Bivariate distributions show the expression of $\gamma \mathrm{H} 2 \mathrm{AX}$ with respect to the cell cycle phase. The percentage of cells with $\gamma \mathrm{H} 2 \mathrm{AX}$ in the entire cell cycle (B) and G2/M phase (C) are shown. Data are presented as the mean \pm SD of the percentage of cells in three independent experiments.

A
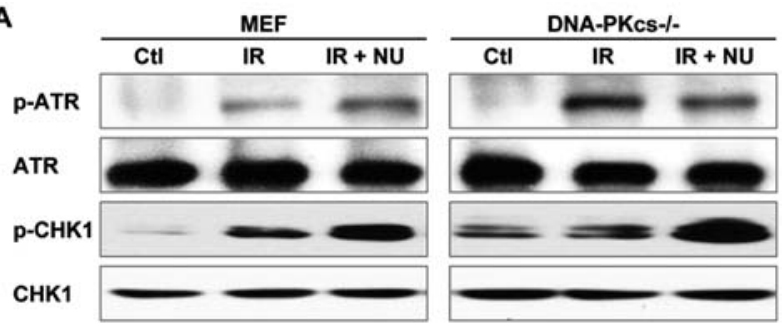

B

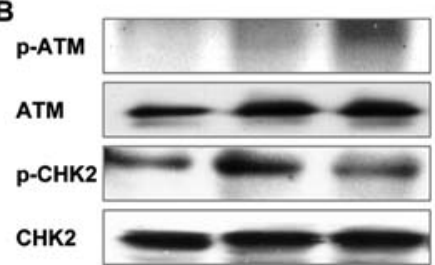

C
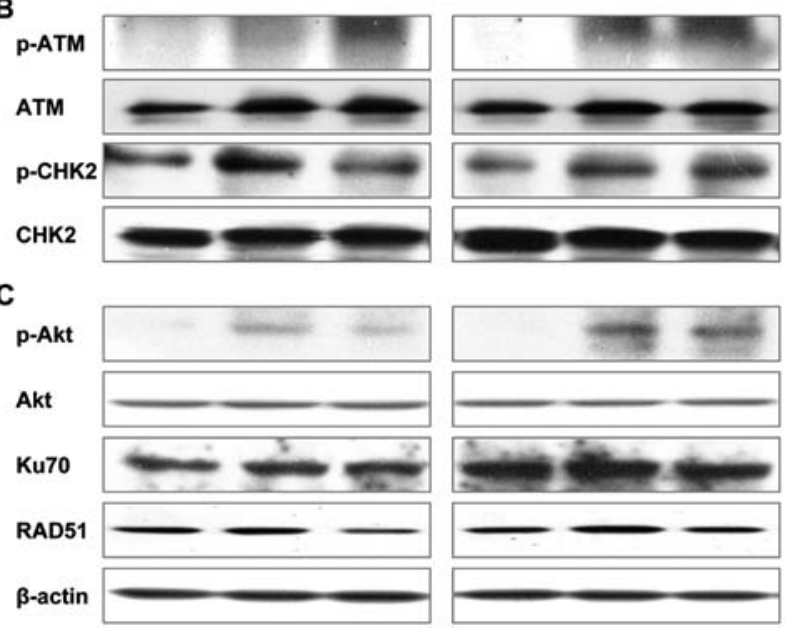

Figure 4. Effect of NU7441 on the activation of crucial DNA damage response proteins. Cells were irradiated with 5 Gy of ionizing radiation in the presence or absence of NU7441 $(2 \mu \mathrm{M})$. The expression of cell cycle checkpoint proteins and double strand break repair proteins was assayed by western blot analysis at $6 \mathrm{~h}$ post-irradiation. combination of IR and NU7441 was statistically significant at 24 and $48 \mathrm{~h}$. The percentage of cells in the G2/M phase at 24 and $48 \mathrm{~h}$ for SUNE1 cells treated with IR and NU7441 was 76 and $65 \%$, respectively; it was 53 and $27 \%$, respectively, for the cells treated with IR alone (Fig. 6A and B).

IR induced cell cycle arrest at $16 \mathrm{~h}$ post-irradiation and then the cell cycle arrest gradually released in IR alone group, but the percentage of SUNE-1 cells in G2/M phase kept at a high level in IR and NU7441 group. Combined treatment of IR and NU7441 did not delay the peak of SUNE-1 cells at G2/M arrest but kept the percentage of cells in G2/M phase at a high level until $48 \mathrm{~h}$ post-irradiation. Comparison of the ratio of G2/M between IR and IR+NU at the same timing is aimed to examine the effect of NU7441 on IR-induced cell cycle arrest.

Next, we investigated the molecular basis in SUNE-1 cells. Levels of phosphorylated CHK1, ATR, CHK2 and ATM were significantly increased in cells treated with IR and NU7441 compared to cells treated with IR alone, indicating that the G2 cell cycle checkpoint is necessary to allow time to repair DNA damage. Treatment with NU7441 attenuated radiationinduced phosphorylation of Akt. The radiation-induced expression of RAD51 was decreased in the presence of NU7441 (Fig. 6C). Taken together, the results indicate that radiosensitive effect of NU7441 is mediated by cell cycle checkpoints by delaying DNA repair, blocking cell cycle progression, and promoting apoptosis. NPC cells are susceptible to combined treatment with NU7441 and irradiation due 


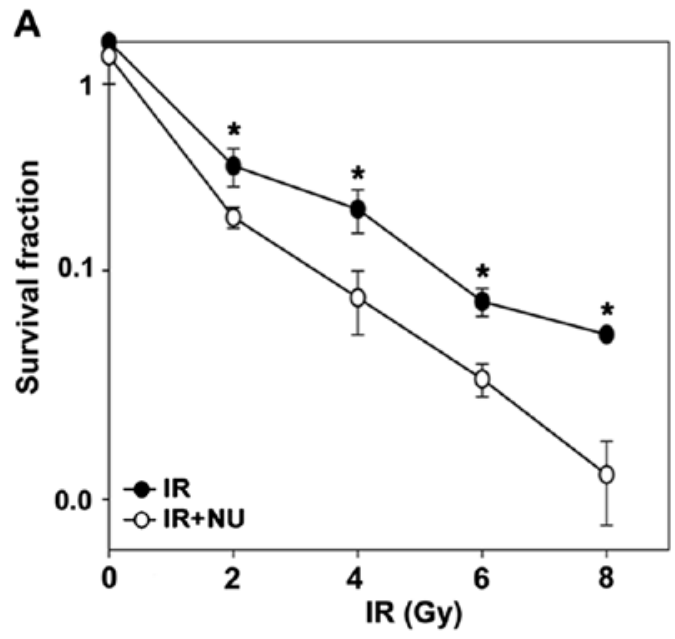

B
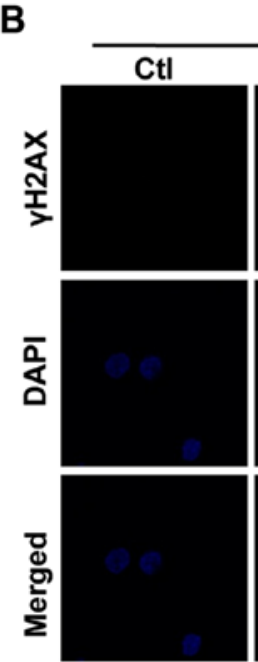

$6 \mathrm{~h}$ post-IR
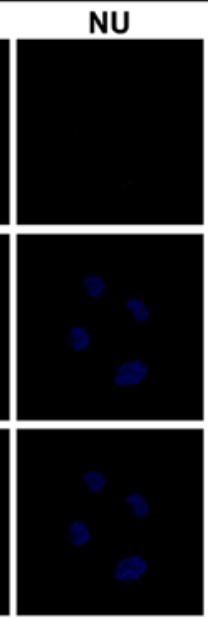
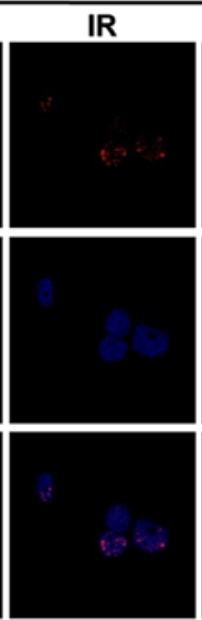

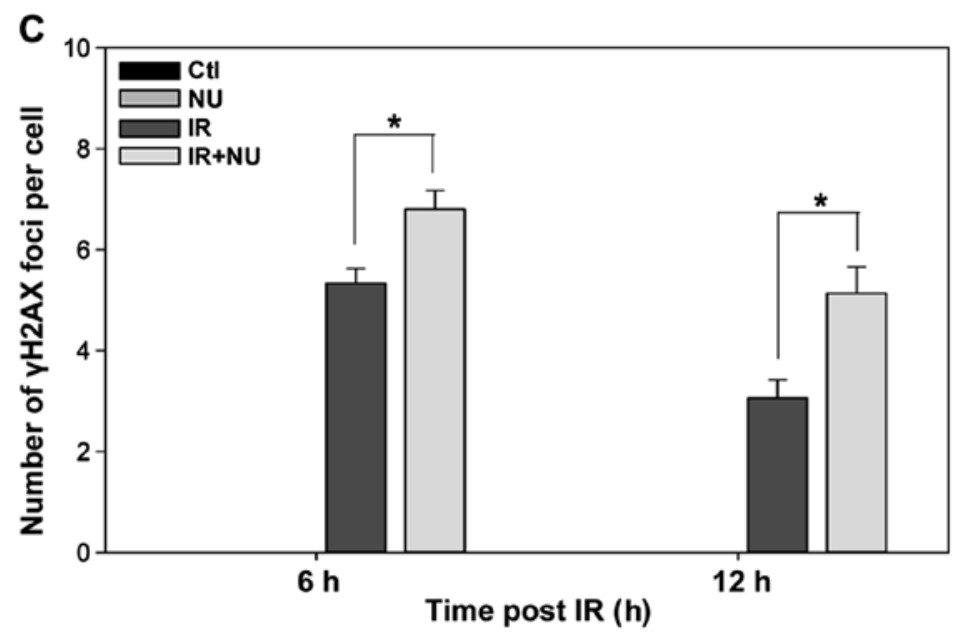

C

$12 \mathrm{~h}$ post-IR

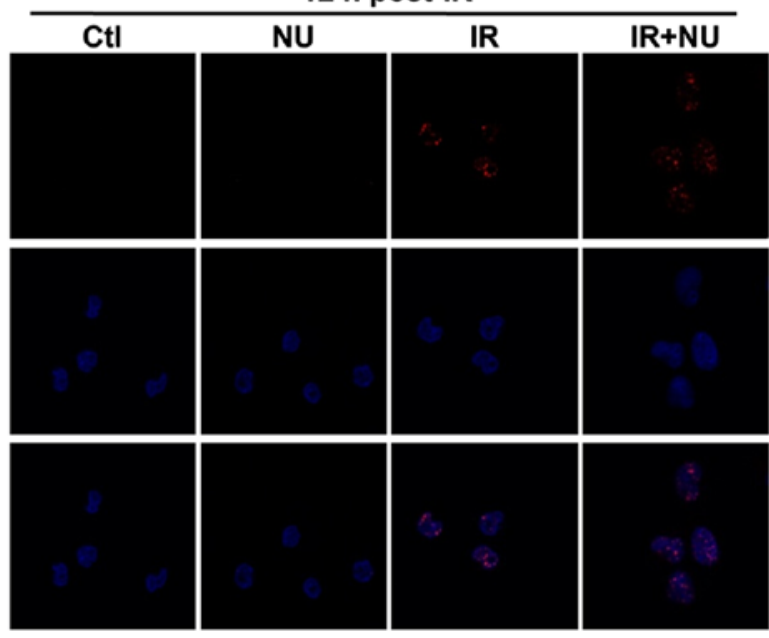

Figure 5. Clonogenic survival and $\gamma \mathrm{H} 2 \mathrm{AX}$ foci formation in nasopharyngeal carcinoma SUNE1 cells with ionizing radiation (IR) and/or NU7441 treatment. (A) SUNE1 cells were exposed to IR alone or in the presence of NU7441 $(1 \mu \mathrm{M})$. Clonogenic survival data are presented as the mean \pm SD. (B) SUNE1 cells were exposed to irradiation of $5 \mathrm{~Gy}$ in the presence or absence of NU7441 for 6 or $12 \mathrm{~h}$ and stained with $\gamma \mathrm{H} 2 \mathrm{AX}$ antibodies and DAPI. Representative photomicrographs (x1,000 magnification) are shown. (C) Quantification of $\gamma \mathrm{H} 2 \mathrm{AX}$ foci formation in (B). " $\mathrm{p}<0.05$, Student's t-test.

to a resulting deficiency in DSB repair and activation of the cell cycle checkpoint. The difference in DSB repair between normal and tumor cells in the suppression of DNA-PK activity offers promise for the development of new radiation-related therapeutic approaches.

\section{Discussion}

Research on DNA double strand break (DSB) repair by nonhomologous end-joining (NHEJ) remains a hot topic in the field of radiation oncology, and it is thought to play a very important role in the therapy of nasopharyngeal carcinoma. DNA-PK is a crucial member of NHEJ. To suppress NHEJ signaling pathway by the DNA-PK inhibitor, NU7441, we explored the synergistic effect on inhibition of DSB repair following exposure to irradiation in NHEJ-competent or -deficient cells and a cancer cell line from a nasopharyngeal carcinoma (NPC) patient.

The present study revealed that: i) a deficiency in DNA-PKcs contributes to the enhancement of radiosensitivity by delaying DSB repair; ii) mechanistically, the combination of IR and NU7441 induces a synergistic effect selectively in NHEJ-competent cells and has a modest effect on DSB repair in cells deficient in DNA-PK activity; and iii) NU7441 enhances the synergistic antitumor effect in NPC cells when applied in combination with irradiation by impairing DSB repair, prolonging cell cycle progression and activating the cell cycle checkpoints, which suggests that DNA-PK inhibitor is a potential radiosensitizer in NPC.

Our previous study isolated DNA-PKcs ${ }^{-/}, \mathrm{Ku}^{-/-}, \mathrm{Ku}^{-1 /}$ MEF cells from relevant gene knockout mice $(3,24,35)$. This study first identified Ku70 as an essential factor in DSB repair and further study demonstrated that decreasing the level of Ku70 results in radiation hypersensitivity $(24,36)$. We designed and generated dominant negative fragment Ku70 (DNKu70). The human cancer cells infected with virus containing $\mathrm{DNKu} 70$ were vulnerable to irradiation (37). Our previous study showed that the radiosensitivity of $\mathrm{Ku}^{-1 /-} \mathrm{MEF}$ cells is similar to that of DNA-PKcs ${ }^{-/-} \mathrm{MEF}$ cells, which is mediated by DSB repair, cell cycle arrest and activation of cell cycle checkpoints (25). Therefore, we only used DNA-PKcs-component and DNA-PKcs knockdown cells to 
A
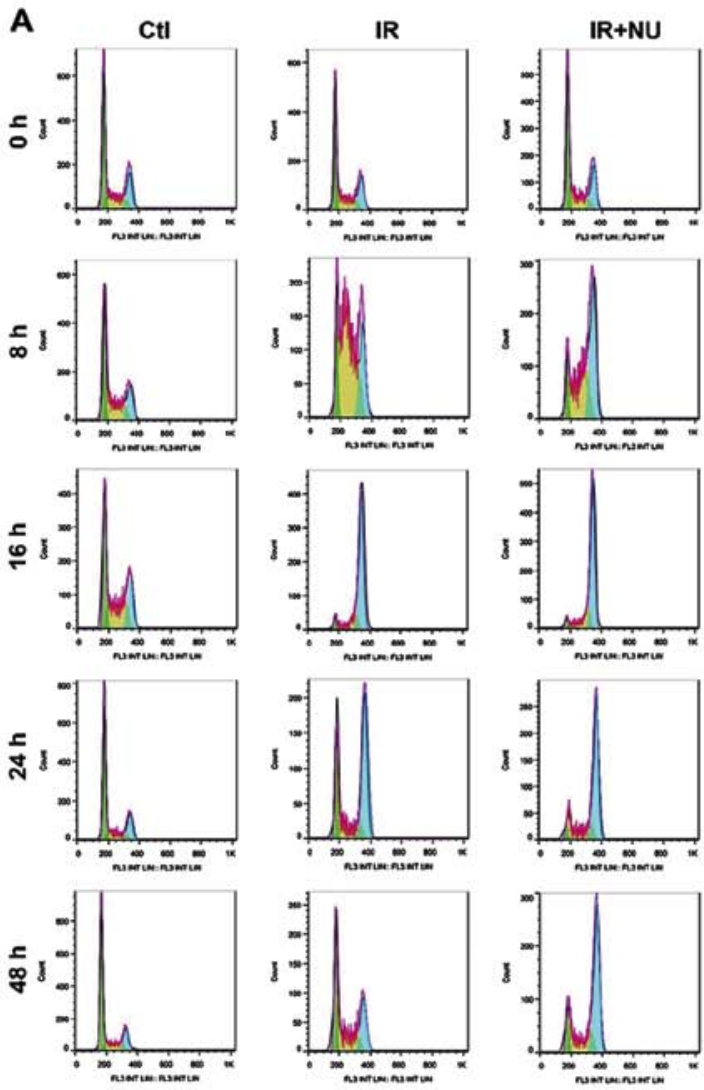

B

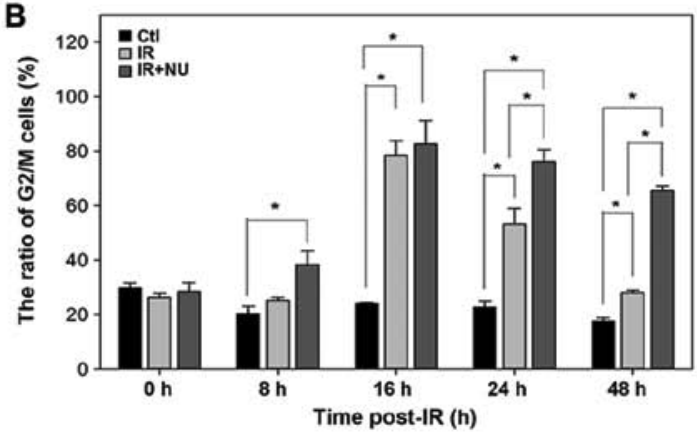

C

P-DNA-PKcs

\begin{tabular}{|c|c|c|c|c|c|}
\hline \multirow[b]{2}{*}{0 Gy } & \multicolumn{2}{|c|}{$6 \mathrm{~h}$} & \multirow[b]{2}{*}{0 Gy } & \multicolumn{2}{|c|}{$12 \mathrm{~h}$} \\
\hline & IR & $\mathrm{IR}+\mathrm{NU}$ & & $\mathbf{I R}$ & $\mathrm{IR}+\mathrm{NU}$ \\
\hline & e. & . & w: & 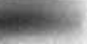 & 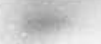 \\
\hline
\end{tabular}

DNA-PKcs

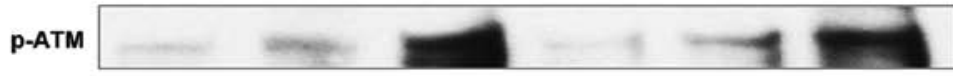

ATM

p-ATR
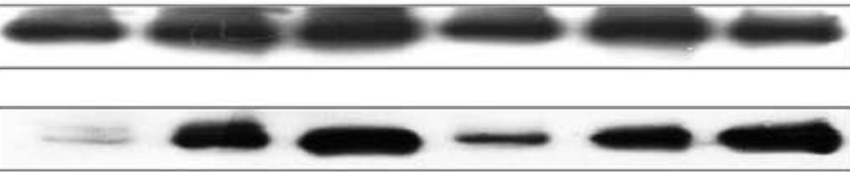

$$
\text { ATR }
$$

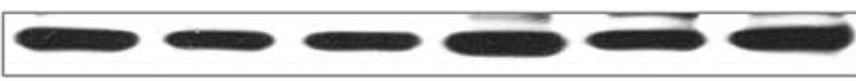

Ku70

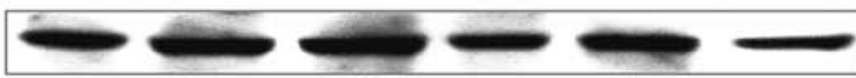

p-CHK1
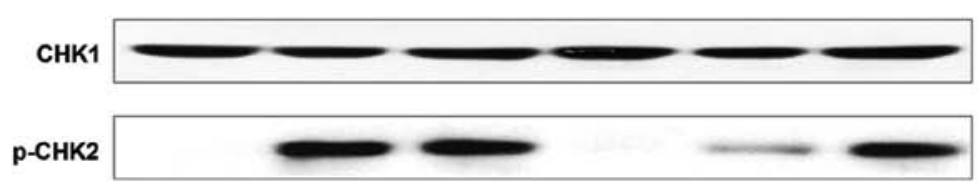

CHK2

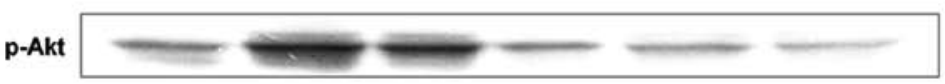

Akt

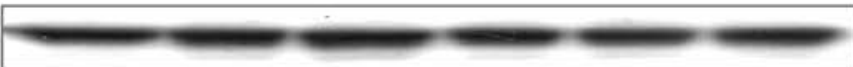

RAD5

$\beta$-actin

Figure 6. Analysis of ionizing radiation (IR)-induced cell cycle distribution and gene expression in the DNA damage response in nasopharyngeal carcinoma SUNE1 cells with IR and/or NU7441 treatment. (A) The cell cycle distribution was determined by FACS flow cytometry of SUNE1 cells at different time intervals with IR (5 Gy) in the presence or absence of NU7441 $(2 \mu \mathrm{M})$. (B) The proportion of G2/M phase was determined. "p $<0.05$ when comparison between groups by ANOVA test. (C) Cells were irradiated with $5 \mathrm{~Gy}$ in the presence or absence of NU7441 $(2 \mu \mathrm{M})$. The expression of cell cycle checkpoint proteins and double strand break repair proteins was assayed by western blot analysis at 6 or $12 \mathrm{~h}$ post-IR.

explore the impact of DNA-PK inhibitor on radiation-induced survival. The results consistently verified that DNA-PKcs ${ }^{-1-}$ MEF cells are more radiosensitive than WT MEF cells and NU7441 enhanced the cytotoxicity in WT MEF cells, but not the DNA-PKcs-deficient cells, implying that it selectively targeted DNA-PK and showed similar radiobiological effect to DNA-PKcs knockout cells. We then explored its enhancement in radiosensitivity in human nasopharyngeal carcinoma cells.

DNA-PK has been shown to be associated with DSB repair and drive cell cycle progression. Silencing of DNA-PKcs by small interfering RNA led to increased radiosensitivity and DSBs (38-40). DNA-PK may coordinate the expression of cell cycle machinery to damage response in regulating mitotic entry (41). Treatment with its small molecular inhibitor significantly increased DSBs in WT MEF cells but did not alter the level of $\gamma \mathrm{H} 2 \mathrm{AX}$ foci in DNA-PK ${ }^{-/}$MEF cells after exposure to IR (Fig. 2). In addition, NU7441 affected the interaction between IR-induced DSBs and G2/M phase (Fig. 3). These data confirmed the crucial role of DNA-PK in response to radiation and demonstrated that cells with individual component of DNA-PK knockdown may not be affected by NU7441 after IR treatment.

Cell cycle checkpoints are biological pathways that provide cells with a mechanism by which to respond to DNA damage by arresting the cell cycle to allow DNA repair. Robust phosphorylation of CHK1 has been reported in cells deficient in DNA-PK (9). We also observed that phosphorylated CHK1 is markedly increased in WT MEF cells treated with IR and NU7441 when compared to cells irradiated alone.

Radiation therapy is one of the important treatment modalities in addition to surgical operation, and chemotherapy for all of those solid cancers. Whereas radiation therapy is the main 
treatment modality in the management of NPC and has achieved excellent outcomes. The 5-year survival rate is $>95 \%$ for patients with early stage disease treated with radiation alone and $60 \%$ for patients with locally advanced disease in combination with chemotherapy. The addition of chemotherapy produced $5-7 \%$ improvement in overall survival when compare to radiation alone. A recent phase 3 clinical trial demonstrated statistically significant survival advantage of the addition of induction chemotherapy (i.e., cisplatin, fluorouracil, and docetaxel; TPF) to concurrent chemo-radiotherapy in patients with locoregionally advanced NPC (42). Whereas surgical operation is not accessible to its proximity of nasopharynx by it complexity with extensive involvement to cranical nerves and skull base. Local cancer recurrence and distant metastasis remains the obstacle for the successful treatment of NPC. Previously we showed that precise radiotherapy is an effective treatment (43), and comprehensive treatment had a favorable prognosis (44). However, local tumor recurrence remains the main obstacle to the successful treatment for patients with locally advanced NPC. Further study will explore the application and mechanism of combined treatment with DNA-PK inhibitor and conventional chemotherapeutic agents in NPC.

Overexpression of Ku70 or DNA-PKcs in NPC patients correlates with a worse clinical outcome, probably because of the capacity to repair DNA damage and to be resistant to radiotherapy (45). For the first time, we have shown that NU7441 can effectively radiosensitize SUNE-1 cells by interfering with DNA repair and delaying the progress through the cell cycle. Combined treatment of IR and NU7441 aggravated G2/M accumulation in SUNE-1 cells. The limitation of the study is that the effect of NU7441 was not examined in normal human cells. Our data provide novel evidence for the application of DNA-PK inhibitor in NPC which needs further investigation. It may be a promising paradigm in the management of NPC.

In conclusion, our study elucidated the functional ramification of DNA-PK in the modification of DSB repair and the cell cycle checkpoints. NU7441 has been shown to significantly enhance radiosensitivity in human NPC cells by antagonizing radiation-induced cellular defense mechanisms, suggesting that DNA-PK inhibitor may enhance the efficacy of radiotherapy in NPC which merits further investigation.

\section{Acknowledgements}

The study was supported in part by National Institutes of Health (grant nos. PO1 CA115675); National Institutes of Health/National Cancer Institute (grant nos. R33 CA109772); National Natural Science Foundation of China (grant nos. 81172209 and 81673088). We thank San Francisco Edit Service for help in English language editing.

\section{References}

1. Liu P, Gan W, Guo C, Xie A, Gao D, Guo J, Zhang J, Willis N, Su A, Asara JM, et al: Akt-mediated phosphorylation of XLF impairs non-homologous end-joining DNA repair. Mol Cell 57: 648-661, 2015.

2. Zhang S, Matsunaga S, Lin YF, Sishc B, Shang Z, Sui J, Shih HY, Zhao Y, Foreman O, Story MD, et al: Spontaneous tumor development in bone marrow-rescued DNA-PKcs (3A/3A) mice due to dysfunction of telomere leading strand deprotection. Oncogene 35: 3909-3918, 2016
3. Kurimasa A, Ouyang H, Dong LJ, Wang S, Li X, Cordon-Cardo C, Chen DJ and Li GC: Catalytic subunit of DNA-dependent protein kinase: Impact on lymphocyte development and tumorigenesis. Proc Natl Acad Sci USA 96: 1403-1408, 1999.

4. Wang S, Guo M, Ouyang H, Li X, Cordon-Cardo C, Kurimasa A, Chen DJ, Fuks Z, Ling CC and Li GC: The catalytic subunit of DNA-dependent protein kinase selectively regulates p53-dependent apoptosis but not cell-cycle arrest. Proc Natl Acad Sci USA 97: 1584-1588, 2000.

5. Chitnis MM, Lodhia KA, Aleksic T, Gao S, Protheroe AS and Macaulay VM: IGF-1R inhibition enhances radiosensitivity and delays double-strand break repair by both non-homologous end-joining and homologous recombination. Oncogene 33: 5262-5273, 2014

6. Lee SH and Kim CH: DNA-dependent protein kinase complex: A multifunctional protein in DNA repair and damage checkpoint. Mol Cells 13: 159-166, 2002.

7. Bunting SF and Nussenzweig A: End-joining, translocations and cancer. Nat Rev Cancer 13: 443-454, 2013.

8. Branzei D and Foiani M: Regulation of DNA repair throughout the cell cycle. Nat Rev Mol Cell Biol 9: 297-308, 2008.

9. Wang X, Li GC, Iliakis G and Wang Y: Ku affects the CHK1dependent $\mathrm{G}$ (2) checkpoint after ionizing radiation. Cancer Res 62: 6031-6034, 2002.

10. Shang ZF, Huang B, Xu QZ, Zhang SM, Fan R, Liu XD, Wang $Y$ and Zhou PK: Inactivation of DNA-dependent protein kinase leads to spindle disruption and mitotic catastrophe with attenuated checkpoint protein 2 Phosphorylation in response to DNA damage. Cancer Res 70: 3657-3666, 2010.

11. Weber AM and Ryan AJ: ATM and ATR as therapeutic targets in cancer. Pharmacol Ther 149: 124-138, 2015.

12. Curtin NJ: DNA repair dysregulation from cancer driver to therapeutic target. Nat Rev Cancer 12: 801-817, 2012.

13. Bouwman P and Jonkers J: The effects of deregulated DNA damage signalling on cancer chemotherapy response and resistance. Nat Rev Cancer 12: 587-598, 2012.

14. Bozulic L, Surucu B, Hynx D and Hemmings BA: PKBalpha/Akt1 acts downstream of DNA-PK in the DNA double-strand break response and promotes survival. Mol Cell 30: 203-213, 2008.

15. Zhao Y, Thomas HD, Batey MA, Cowell IG, Richardson CJ, Griffin RJ, Calvert AH, Newell DR, Smith GC and Curtin NJ: Preclinical evaluation of a potent novel DNA-dependent protein kinase inhibitor NU7441. Cancer Res 66: 5354-5362, 2006.

16. Yu L, Tumati V, Tseng SF, Hsu FM, Kim DN, Hong D, Hsieh JT, Jacobs C, Kapur P and Saha D: DAB2IP regulates autophagy in prostate cancer in response to combined treatment of radiation and a DNA-PKcs inhibitor. Neoplasia 14: 1203-1212, 2012.

17. Tavecchio M, Munck JM, Cano C, Newell DR and Curtin NJ: Further characterisation of the cellular activity of the DNA-PK inhibitor, NU7441, reveals potential cross-talk with homologous recombination. Cancer Chemother Pharmacol 69: 155-164, 2012.

18. Javvadi P, Makino H, Das AK, Lin YF, Chen DJ, Chen BP and Nirodi CS: Threonine 2609 phosphorylation of the DNA-dependent protein kinase is a critical prerequisite for epidermal growth factor receptor-mediated radiation resistance. Mol Cancer Res 10: 1359-1368, 2012.

19. Ciszewski WM, Tavecchio M, Dastych J and Curtin NJ: DNA-PK inhibition by NU7441 sensitizes breast cancer cells to ionizing radiation and doxorubicin. Breast Cancer Res Treat 143: 47-55, 2014.

20. Tichy A, Durisova K, Salovska B, Pejchal J, Zarybnicka L, Vavrova J, Dye NA and Sinkorova Z: Radio-sensitization of human leukaemic MOLT-4 cells by DNA-dependent protein kinase inhibitor, NU7441. Radiat Environ Biophys 53: 83-92, 2014.

21. Cornell L, Munck JM, Alsinet C, Villanueva A, Ogle L, Willoughby CE, Televantou D, Thomas HD, Jackson J, Burt AD, et al: DNA-PK-A candidate driver of hepatocarcinogenesis and tissue biomarker that predicts response to treatment and survival. Clin Cancer Res 21: 925-933, 2015.

22. Yu L, Shang ZF, Hsu FM, Zhang Z, Tumati V, Lin YF, Chen BP and Saha D: NSCLC cells demonstrate differential mode of cell death in response to the combined treatment of radiation and a DNA-PKcs inhibitor. Oncotarget 6: 3848-3860, 2015.

23. Chua MLK, Wee JTS, Hui EP and Chan ATC: Nasopharyngeal carcinoma. Lancet 387: 1012-1024, 2016.

24. Ouyang H, Nussenzweig A, Kurimasa A, Soares VC, Li X, Cordon-Cardo C, Li W, Cheong N, Nussenzweig M, Iliakis G, et al: $\mathrm{Ku} 70$ is required for DNA repair but not for $\mathrm{T}$ cell antigen receptor gene recombination in vivo. J Exp Med 186: 921-929, 1997. 
25. Dong J, Zhang T, Ren Y, Wang Z, Ling CC, He F, Li GC, Wang $C$ and Wen B: Inhibiting DNA-PKes in a non-homologous end-joining pathway in response to DNA double-strand breaks. Oncotarget 8: 22662-22673, 2017.

26. Lu QP, Chen WD, Peng JR, Xu YD, Cai Q, Feng GK, Ding K, Zhu XF and Guan Z: Antitumor activity of $7 \mathrm{RH}$, a discoidin domain receptor 1 inhibitor, alone or in combination with dasatinib exhibits antitumor effects in nasopharyngeal carcinoma cells. Oncol Lett 12: 3598-3608, 2016.

27. Zhao M, Luo R, Liu Y, Gao L, Fu Z, Fu Q, Luo X, Chen Y, Deng X, Liang $\mathrm{Z}$, et al: miR-3188 regulates nasopharyngeal carcinoma proliferation and chemosensitivity through a FOXO1-modulated positive feedback loop with mTOR-p-PI3K/AKT-c-JUN. Nat Commun 7: 11309, 2016.

28. Wen B, Deutsch E, Marangoni E, Frascona V, Maggiorella L, Abdulkarim B, Chavaudra N and Bourhis J: Tyrphostin AG 1024 modulates radiosensitivity in human breast cancer cells. Br J Cancer 85: 2017-2021, 2001.

29. Kataoka Y, Bindokas VP, Duggan RC, Murley JS and Grdina DJ: Flow cytometric analysis of phosphorylated histone H2AX following exposure to ionizing radiation in human microvascular endothelial cells. J Radiat Res (Tokyo) 47: 245-257, 2006.

30. Cuneo KC, Morgan MA, Davis MA, Parcels LA, Parcels J, Karnak D, Ryan C, Liu N, Maybaum J and Lawrence TS Weel kinase inhibitor AZD1775 radiosensitizes hepatocellular carcinoma regardless of TP53 mutational status through induction of replication stress. Int J Radiat Oncol Biol Phys 95: 782-790, 2016

31. Bonner WM, Redon CE, Dickey JS, Nakamura AJ Sedelnikova OA, Solier S and Pommier Y: GammaH2AX and cancer. Nat Rev Cancer 8: 957-967, 2008.

32. Sulli G, Di Micco R and d'Adda di Fagagna F: Crosstalk between chromatin state and DNA damage response in cellular senescence and cancer. Nat Rev Cancer 12: 709-720, 2012.

33. Zhou XY, Wang X, Wang H, Chen DJ, Li GC, Iliakis G and Wang Y: Ku affects the ATM-dependent S phase checkpoint following ionizing radiation. Oncogene 21: 6377-6381, 2002.

34. Morgan MA and Lawrence TS: Molecular pathways: Overcoming radiation resistance by targeting DNA damage response pathways. Clin Cancer Res 21: 2898-2904, 2015.

35. Nussenzweig A, Chen C, da Costa Soares V, Sanchez M, Sokol K, Nussenzweig MC and Li GC: Requirement for Ku80 in growth and immunoglobulin V (D)J recombination. Nature 382: 551-555, 1996

36. Li GC, He F, Shao X, Urano M, Shen L, Kim D, Borrelli M, Leibel SA, Gutin PH and Ling CC: Adenovirus-mediated heatactivated antisense $\mathrm{Ku} 70$ expression radiosensitizes tumor cells in vitro and in vivo. Cancer Res 63: 3268-3274, 2003.
37. He F, Li L, Kim D, Wen B, Deng X, Gutin PH, Ling CC and Li GC: Adenovirus-mediated expression of a dominant negative $\mathrm{Ku} 70$ fragment radiosensitizes human tumor cells under aerobic and hypoxic conditions. Cancer Res 67: 634-642, 2007.

38. Peng Y, Zhang Q, Nagasawa H, Okayasu R, Liber HL and Bedford JS: Silencing expression of the catalytic subunit of DNA-dependent protein kinase by small interfering RNA sensitizes human cells for radiation-induced chromosome damage, cell killing, and mutation. Cancer Res 62: 6400-6404, 2002.

39. An J, Xu QZ, Sui JL, Bai B and Zhou PK: Downregulation of c-myc protein by siRNA-mediated silencing of DNA-PKcs in HeLa cells. Int J Cancer 117: 531-537, 2005.

40. Toulany M, Kehlbach R, Florczak U, Sak A, Wang S, Chen J, Lobrich M and Rodemann HP: Targeting of AKT1 enhances radiation toxicity of human tumor cells by inhibiting DNA-PKcsdependent DNA double-strand break repair. Mol Cancer Ther 7: 1772-1781, 2008

41. Goodwin JF and Knudsen KE: Beyond DNA repair: DNA-PK function in cancer. Cancer Discov 4: 1126-1139, 2014.

42. Sun Y, Li WF, Chen NY, Zhang N, Hu GQ, Xie FY, Sun Y, Chen XZ, Li JG, Zhu XD, et al: Induction chemotherapy plus concurrent chemoradiotherapy versus concurrent chemoradiotherapy alone in locoregionally advanced nasopharyngeal carcinoma: A phase 3, multicentre, randomised controlled trial. Lancet Oncol 17: 1509-1520, 2016.

43. Ren Y, Zhao Q, Liu H, Huang Y, Wang Z, Cao X, Teh BS and Wen B: 3D-image-guided HDR-brachytherapy versus 2D HDR brachytherapy after external beam radiotherapy for early T-stage nasopharyngeal carcinoma. BMC Cancer 14: 894, 2014.

44. Wang C, Huang P, Guo L, Wang Z, Zhang T, Dong J, Shi J, Li Y, Guo X, Cao K, et al: Prognostic analysis in patients with metastatic nasopharyngeal carcinoma at initial diagnosis. Jacobs J Radiat Oncol 1: 10, 2014.

45. Lee SW, Cho KJ, Park JH, Kim SY, Nam SY, Lee BJ, Kim SB Choi SH, Kim JH, Ahn SD, et al: Expressions of Ku70 and DNA-PKes as prognostic indicators of local control in nasopharyngeal carcinoma. Int J Radiat Oncol Biol Phys 62: 1451-1457, 2005.

c) (†) $\Theta$ This work is licensed under a Creative Commons Attribution-NonCommercial-NoDerivatives 4.0 International (CC BY-NC-ND 4.0) License. 